Applied Mathematical Sciences, Vol. 8, 2014, no. 166, 8309 - 8315

HIKARI Ltd, www.m-hikari.com

http://dx.doi.org/10.12988/ams.2014.411918

\title{
A Laplace Type Problem for a Lattice of Rectangles with Triangular Obstacles
}

\author{
Uwe Bäsel* and Andrei Duma** \\ ${ }^{*}$ HTWK Leipzig \\ Fakultät Maschinenbau und Energietechnik \\ 04251 Leipzig, Germany \\ **FernUniversität in Hagen \\ Fakultät Mathematik und Informatik \\ 58084 Hagen, Germany \\ Dedicated to Professor Marius Stoka on the occasion of his $80^{\text {th }}$ birthday \\ Copyright (c) 2014 Uwe Bäsel and Andrei Duma. This is an open access article dis- \\ distribution, and reproduction in any medium, provided the original work is properly cited.
} tributed under the Creative Commons Attribution License, which permits unrestricted use,

\begin{abstract}
In this paper, we calculate the probability that a small rectangle hits a lattice of rectangles with triangular obstacles.
\end{abstract}

Mathematics Subject Classification: 60D05, 52A22

Keywords: Random convex sets, hitting probabilities, intersection probabilities, Laplace type problem, lattice of rectangles, obstacles

\section{Introduction}

A rectangle $\mathcal{D}$ with side lengths $L$ and $l, L \geq l$, is randomly thrown onto a plane lattice $\mathcal{R}_{a, b, m}$ of rectangles with side lengths $4 a$ and $b$, and $2 a$ and $b$, respectively (see Fig. 1). In every corner of the rectangles there is an obstacle that is an isosceles right triangle with legs of length $m / 2$ and hypotenuse of length $m / \sqrt{2}$ with $m<\min (2 a, b)$. 


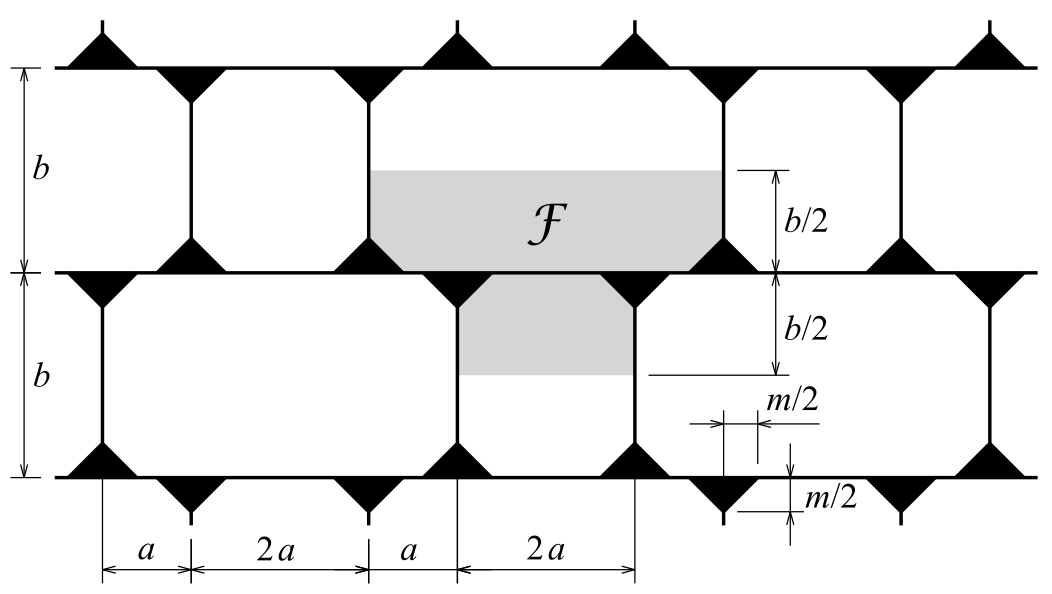

Fig. 1: Lattice $\mathcal{R}_{a, b, m}$ with its fundamental cell $\mathcal{F}$

The fundamental cell $\mathcal{F}$ of $\mathcal{R}_{a, b, m}$ is shaded in Fig. 1. Using the Cartesian coordinate system in Fig. 2, we have

$$
\begin{aligned}
\mathcal{F}= & \left\{(x, y) \in \mathbb{R}^{2} \mid-2 a \leq x \leq 2 a, 0 \leq y \leq b / 2\right\} \cup \\
& \left\{(x, y) \in \mathbb{R}^{2} \mid-a \leq x \leq a,-b / 2 \leq y \leq 0\right\} .
\end{aligned}
$$

The area of $\mathcal{F}$ is equal to $3 a b$. After attaching a fixed reference point $C$ to $\mathcal{D}$, we define the random throw of $\mathcal{D}$ onto $\mathcal{R}_{a, b, m}$ as follows: The coordinates $x$ and $y$ of $C$ are random variables uniformly distributed in $[-2 a, 2 a],[-b / 2, b / 2]$, respectively; the angle $\phi$ between the $x$-axis and the longer sides of $\mathcal{D}$ is a random variable uniformly distributed in $[0,2 \pi]$. All three random variables are stochastically independent. We only consider events with $C \in \mathcal{F}$.

In the following, we assume $\mathcal{D}$ to be small with respect to $\mathcal{R}_{a, b, m}$; this means

$$
L \leq \min (2 a-m, b-m, m / \sqrt{2}) .
$$

We denote by $d=\sqrt{L^{2}+l^{2}}$ the length of the diagonals of $\mathcal{D}$, and by $\alpha$ the angle between a diagonal and the longer sides of $\mathcal{D}$, hence $\sin \alpha=l / d$ and $\cos \alpha=L / d$.

Known results for lattices with obstacles are for instance to be found in [1], [2], [3] and [4]. 


\section{Hitting probability}

Theorem 2.1. If inequality (1) holds, then the probability $p$ that $\mathcal{D}$ hits $\mathcal{R}_{a, b, m}$ is given by

$$
\begin{aligned}
p=\frac{1}{6 \pi a b}[ & 4(3 a+b)(L+l)-4\left(L^{2}+l^{2}\right)+\pi(L-l)^{2} \\
& \left.-4(2-\sqrt{2}) m(L+l)+\pi m^{2}\right] .
\end{aligned}
$$

Proof. As reference point $C$ of $\mathcal{D}$ we choose its barycentre. Due to the symmetry of $\mathcal{F}$ with respect to the $y$-axis, it is sufficient to consider $\phi$ only in the interval $[0, \pi / 2]$ in order to get all positions of $\mathcal{D}$ exactly once. Let $\mathcal{F}(\mathcal{D}, \phi)$ be the set of all points $(x, y) \in \mathcal{F}$ where the rectangle $\mathcal{D}$ with coordinates $(x, y)$ and angle $\phi$ does not intersect the lattice $\mathcal{R}_{a, b, m}$. Let $\mathcal{M}$ be the set of all rectangles $\mathcal{D}$ with barycentre in $\mathcal{F}$, and $\mathcal{N} \subset \mathcal{M}$ the set of all rectangles $\mathcal{D}$ with barycentre in $\mathcal{F}$, not intersecting $\mathcal{R}_{a, b, m}$. Then, the probability $p$ is given by Stoka's formula

$$
p=1-\frac{\mu(\mathcal{N})}{\mu(\mathcal{M})},
$$

where $\mu$ is the Lebesgue measure in the Euclidean plane. We compute $\mu(\mathcal{M})$ and $\mu(\mathcal{N})$ by means of the elementary kinematic density $\mathrm{d} x \wedge \mathrm{d} y \wedge \mathrm{d} \phi=\mathrm{d} K$ for the group of motions in the plane (see [5, pp. 126-128], [6, p. 85]), and get

$$
\begin{aligned}
& \mu(\mathcal{M})=\int_{0}^{\pi / 2} \mathrm{~d} \phi \iint_{(x, y) \in \mathcal{F}} \mathrm{d} x \mathrm{~d} y=\frac{\pi}{2} \text { area } \mathcal{F}=\frac{3 \pi a b}{2}, \\
& \mu(\mathcal{N})=\int_{0}^{\pi / 2} \mathrm{~d} \phi \iint_{(x, y) \in \mathcal{F}(\mathcal{D}, \phi)} \mathrm{d} x \mathrm{~d} y=\int_{0}^{\pi / 2} \operatorname{area} \mathcal{F}(\mathcal{D}, \phi) \mathrm{d} \phi
\end{aligned}
$$

hence

$$
p=1-\frac{2}{3 \pi a b} \int_{0}^{\pi / 2} \text { area } \mathcal{F}(\mathcal{D}, \phi) \mathrm{d} \phi .
$$

Therefore, for the calculation of $p$ it is sufficient to know the continuous function $[0, \pi / 2] \rightarrow \mathbb{R}, \phi \mapsto$ area $\mathcal{F}(\mathcal{D}, \phi)$, and to integrate it from 0 to $\pi / 2$ with respect to $\phi$. The set $\mathcal{F}(\mathcal{D}, \phi)$ consists of two hexagons. In order to calculate the dimensions of these hexagons, we have to distinguish the cases $\phi \in[0, \pi / 4)$ and $\phi \in[\pi / 4, \pi / 2]$, see Figures 2 and 3 .

In the case $\phi \in[0, \pi / 4), \mathcal{F}(\mathcal{D}, \phi)$ consists of

a) two congruent trapezia $t(\phi)$ with parallel sides of lengths

$$
\frac{b}{2}-\frac{d}{2} \sin (\alpha+\phi) \text { and } \frac{b}{2}-\frac{d}{2} \sin (\alpha+\phi)-\left(\frac{m}{2}-l \sin \phi\right),
$$

and distance $(m / 2)-l \sin \phi$, 
b) two congruent trapezia $T(\phi)$ with parallel sides of lengths

$$
\frac{b}{2}-\frac{d}{2} \sin (\alpha+\phi) \text { and } \quad \frac{b}{2}-\frac{d}{2} \sin (\alpha+\phi)-\left(\frac{m}{2}-L \sin \phi\right),
$$

and distance $(m / 2)-L \sin \phi$,

c) one rectangle $\Delta_{4 a}(\phi)$ with side lengths

$$
4 a-m-L(\cos \phi-\sin \phi) \quad \text { and } \quad \frac{b}{2}-\frac{d}{2} \sin (\alpha+\phi),
$$

and one rectangle $\Delta_{2 a}(\phi)$ with side lengths

$$
2 a-m-L(\cos \phi-\sin \phi) \quad \text { and } \quad \frac{b}{2}-\frac{d}{2} \sin (\alpha+\phi) .
$$

Therefore, we have

$$
\begin{aligned}
& \text { area } \mathcal{F}(\mathcal{D}, \phi) \\
& \begin{aligned}
= & \cdot \frac{1}{2}\left[b-d \sin (\alpha+\phi)-\left(\frac{m}{2}-l \sin \phi\right)\right]\left(\frac{m}{2}-l \sin \phi\right) \\
& +2 \cdot \frac{1}{2}\left[b-d \sin (\alpha+\phi)-\left(\frac{m}{2}-L \sin \phi\right)\right]\left(\frac{m}{2}-L \sin \phi\right) \\
& +[6 a-2 m-2 L(\cos \phi-\sin \phi)]\left[\frac{b}{2}-\frac{d}{2} \sin (\alpha+\phi)\right] \\
= & 3 a b-\frac{m^{2}}{2}-3 a d \sin (\alpha+\phi)-b l \sin \phi-b L \cos \phi+l m \sin \phi+L m \sin \phi \\
& +d l \sin (\alpha+\phi) \sin \phi+d L \sin (\alpha+\phi) \cos \phi-\left(l^{2}+L^{2}\right) \sin ^{2} \phi,
\end{aligned}
\end{aligned}
$$

hence

$$
\begin{aligned}
& \int_{0}^{\pi / 4} \operatorname{area} \mathcal{F}(\mathcal{D}, \phi) \mathrm{d} \phi \\
& =\frac{\pi}{4}\left(3 a b-\frac{m^{2}}{2}\right)-3\left(\cos \alpha-\frac{1}{\sqrt{2}} \cos \alpha+\frac{1}{\sqrt{2}} \sin \alpha\right) a d \\
& \quad+\left(1-\frac{\sqrt{2}}{2}\right) b l-\frac{\sqrt{2}}{2} b L+\left(1-\frac{\sqrt{2}}{2}\right) l m+\left(1-\frac{\sqrt{2}}{2}\right) L m \\
& \quad+\left(\frac{\pi}{8} \cos \alpha-\frac{1}{4} \cos \alpha+\frac{1}{4} \sin \alpha\right) d l+\left(\frac{\pi}{8} \sin \alpha+\frac{1}{4} \sin \alpha+\frac{1}{4} \cos \alpha\right) d L \\
& \quad-\left(\frac{\pi}{8}-\frac{1}{4}\right)\left(l^{2}+L^{2}\right) .
\end{aligned}
$$




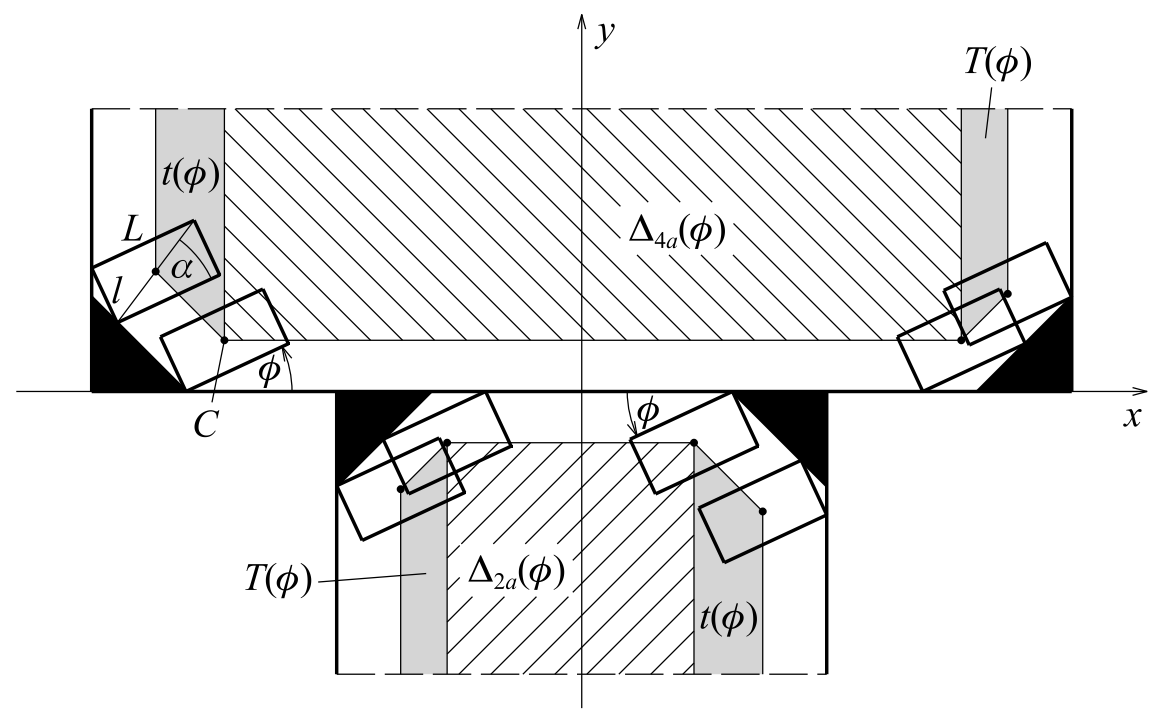

Fig. 2: Case $\phi \in[0, \pi / 4)$

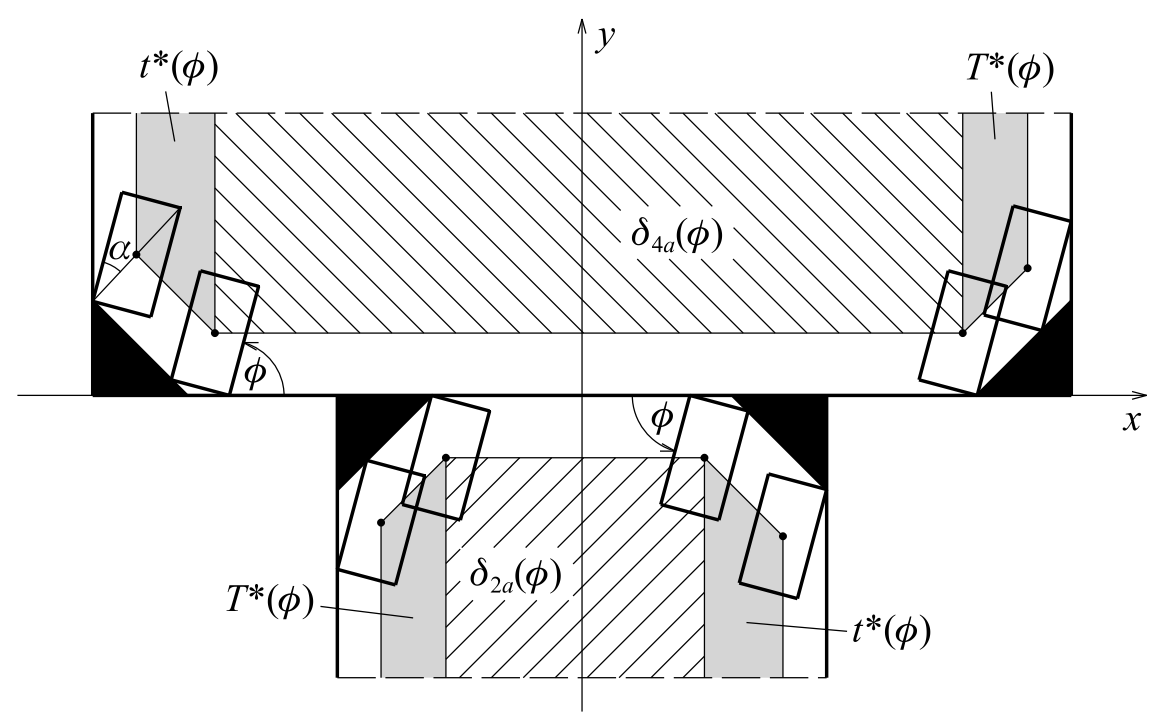

Fig. 3: Case $\phi \in[\pi / 4, \pi / 2]$

In the case $\phi \in[\pi / 4, \pi / 2], \mathcal{F}(\mathcal{D}, \phi)$ is the union of the following polygons (see Fig. 3):

a) two congruent trapezia $t^{*}(\phi)$ with parallel sides of lengths

$$
\frac{b}{2}-\frac{d}{2} \sin (\alpha+\phi) \text { and } \frac{b}{2}-\frac{d}{2} \sin (\alpha+\phi)-\left(\frac{m}{2}-l \cos \phi\right),
$$

and distance $(m / 2)-l \cos \phi$,

b) two congruent trapezia $T^{*}(\phi)$ with parallel sides of lengths

$$
\frac{b}{2}-\frac{d}{2} \sin (\alpha+\phi) \text { and } \frac{b}{2}-\frac{d}{2} \sin (\alpha+\phi)-\left(\frac{m}{2}-l \cos \phi\right),
$$


and distance $(m / 2)-L \cos \phi$,

c) one rectangle $\delta_{4 a}(\phi)$ with side lengths

$$
4 a-m-l(\sin \phi-\cos \phi) \text { and } \frac{b}{2}-\frac{d}{2} \sin (\alpha+\phi),
$$

and one rectangle $\delta_{2 a}(\phi)$ with side lengths

$$
2 a-m-l(\sin \phi-\cos \phi) \text { and } \frac{b}{2}-\frac{d}{2} \sin (\alpha+\phi) \text {. }
$$

Hence

$$
\begin{aligned}
& \text { area } \mathcal{F}(\mathcal{D}, \phi) \\
& \begin{aligned}
= & \cdot \frac{1}{2}\left[b-d \sin (\alpha+\phi)-\left(\frac{m}{2}-l \cos \phi\right)\right]\left(\frac{m}{2}-l \cos \phi\right) \\
& +2 \cdot \frac{1}{2}\left[b-d \sin (\alpha+\phi)-\left(\frac{m}{2}-L \cos \phi\right)\right]\left(\frac{m}{2}-L \cos \phi\right) \\
& +[6 a-2 m-2 l(\sin \phi-\cos \phi)]\left[\frac{b}{2}-\frac{d}{2} \sin (\alpha+\phi)\right] \\
= & 3 a b-\frac{m^{2}}{2}-3 a d \sin (\alpha+\phi)-b l \sin \phi-b L \cos \phi+l m \cos \phi+L m \cos \phi \\
& +d l \sin (\alpha+\phi) \sin \phi+d L \sin (\alpha+\phi) \cos \phi-\left(l^{2}+L^{2}\right) \cos ^{2} \phi .
\end{aligned}
\end{aligned}
$$

As plausibility check, from (4) and (6) one finds that area $\mathcal{F}(\mathcal{D}, \cdot)$ is continuous in $\phi=\pi / 4$ with

$$
\begin{aligned}
& \text { area } \mathcal{F}(\mathcal{D}, \pi / 4) \\
& =3 a b-\frac{m^{2}}{2}-3 a d \sin \left(\alpha+\frac{\pi}{4}\right)-\frac{\sqrt{2}}{2} b(l+L)+\frac{\sqrt{2}}{2} m(l+L) \\
& \quad+\frac{\sqrt{2}}{2} d(l+L) \sin \left(\alpha+\frac{\pi}{4}\right)-\frac{1}{2}\left(l^{2}+L^{2}\right) .
\end{aligned}
$$

Now we get

$$
\begin{aligned}
& \int_{\pi / 4}^{\pi / 2} \operatorname{area} \mathcal{F}(\mathcal{D}, \phi) \mathrm{d} \phi \\
& =\frac{\pi}{4}\left(3 a b-\frac{m^{2}}{2}\right)-3\left(\sin \alpha-\frac{1}{\sqrt{2}} \sin \alpha+\frac{1}{\sqrt{2}} \cos \alpha\right) a d \\
& \quad-\frac{\sqrt{2}}{2} b l-\left(1-\frac{\sqrt{2}}{2}\right) b L+\left(1-\frac{\sqrt{2}}{2}\right) l m+\left(1-\frac{\sqrt{2}}{2}\right) L m \\
& \quad+\left(\frac{\pi}{8} \cos \alpha+\frac{1}{4} \cos \alpha+\frac{1}{4} \sin \alpha\right) d l+\left(\frac{\pi}{8} \sin \alpha-\frac{1}{4} \sin \alpha+\frac{1}{4} \cos \alpha\right) d L \\
& \quad-\left(\frac{\pi}{8}-\frac{1}{4}\right)\left(l^{2}+L^{2}\right) .
\end{aligned}
$$


With $d \sin \alpha=l$ and $d \cos \alpha=L$, from (5) and (7) it follows that

$$
\begin{aligned}
\int_{0}^{\pi / 2} \operatorname{area} \mathcal{F}(\mathcal{D}, \phi) \mathrm{d} \phi= & \frac{\pi}{2}\left(3 a b-\frac{m^{2}}{2}\right)-(3 a+b)(L+l) \\
& +(2-\sqrt{2}) m(L+l)+L^{2}+l^{2}-\frac{\pi}{4}(L-l)^{2} .
\end{aligned}
$$

The application of formula (3) completes the proof.

Remark 1. The probability $p$ that a needle of length

$$
L \leq \min \left(2 a-m, b-m, \frac{m}{\sqrt{2}}\right)
$$

hits $\mathcal{R}_{a, b, m}$ is given by

$$
p=\frac{1}{6 \pi a b}\left[4(3 a+b) L-(4-\pi) L^{2}-4(2-\sqrt{2}) m L+\pi m^{2}\right] .
$$

Remark 2. From (2) and (8) it follows that in the set of all rectangles with

constant perimeter $u=2(l+L)$, the maximum of $\int_{0}^{\pi / 2}$ area $\mathcal{F}(\mathcal{D}, \phi) \mathrm{d} \phi$ and hence the minimum of $p$ is obtained only for squares with side length $u / 4$.

Acknowledgement. The authors would like to thank Prof. Marius Stoka who suggested to consider the lattice $\mathcal{R}_{a, b, m}$.

\section{References}

[1] G. Caristi, M. Stoka, Laplace problems for regular lattices with an even number of different obstacles, Österreichische Akademie der Wissenschaften, Sitzungsber. Abt. II, 219 (2010), 3-11.

[2] G. Caristi, M. Stoka, Some extensions of the Laplace problem, Rend. Circ. Mat. Palermo 60 (2011), 89-98.

[3] A. Duma, M. Stoka, Geometric probabilities for quadratic lattices with quadratic obstacles, Annales de l'I.S.U.P. 48, No. 1-2 (2004), 19-24.

[4] A. Duma, M. Stoka, Geometric probabilities for triangular lattices with triangular obstacles, Annales de l'I.S.U.P. 49, No. 2-3 (2005), 57-72.

[5] H. Poincaré, Calcul de Probabilités, 2nd ed., Gauthier-Villars, Paris, 1912.

[6] L. A. Santaló, Integral Geometry and Geometric Probability, AddisonWesley, London, 1976.

Received: September 1, 2014; Published: November 25, 2014 